\title{
EXTRA-TERRESTRIAL LIFE IN THE MILKY WAY GALAXY?
}

_ Neal J. Evans II - University of Texas at Austin - DOI: 10.1051/epn/2014503

Are we alone? Along with questions about black holes, this is one of the questions most commonly asked of astrophysicists. While the simple answer is that we don't know, logical and rational exploration of the question can be illuminating and a very good way to introduce non-scientists to the "scientific worldview." This short essay is based on a class for students not majoring in science, which I have taught for nearly forty years at the University of Texas at Austin. 


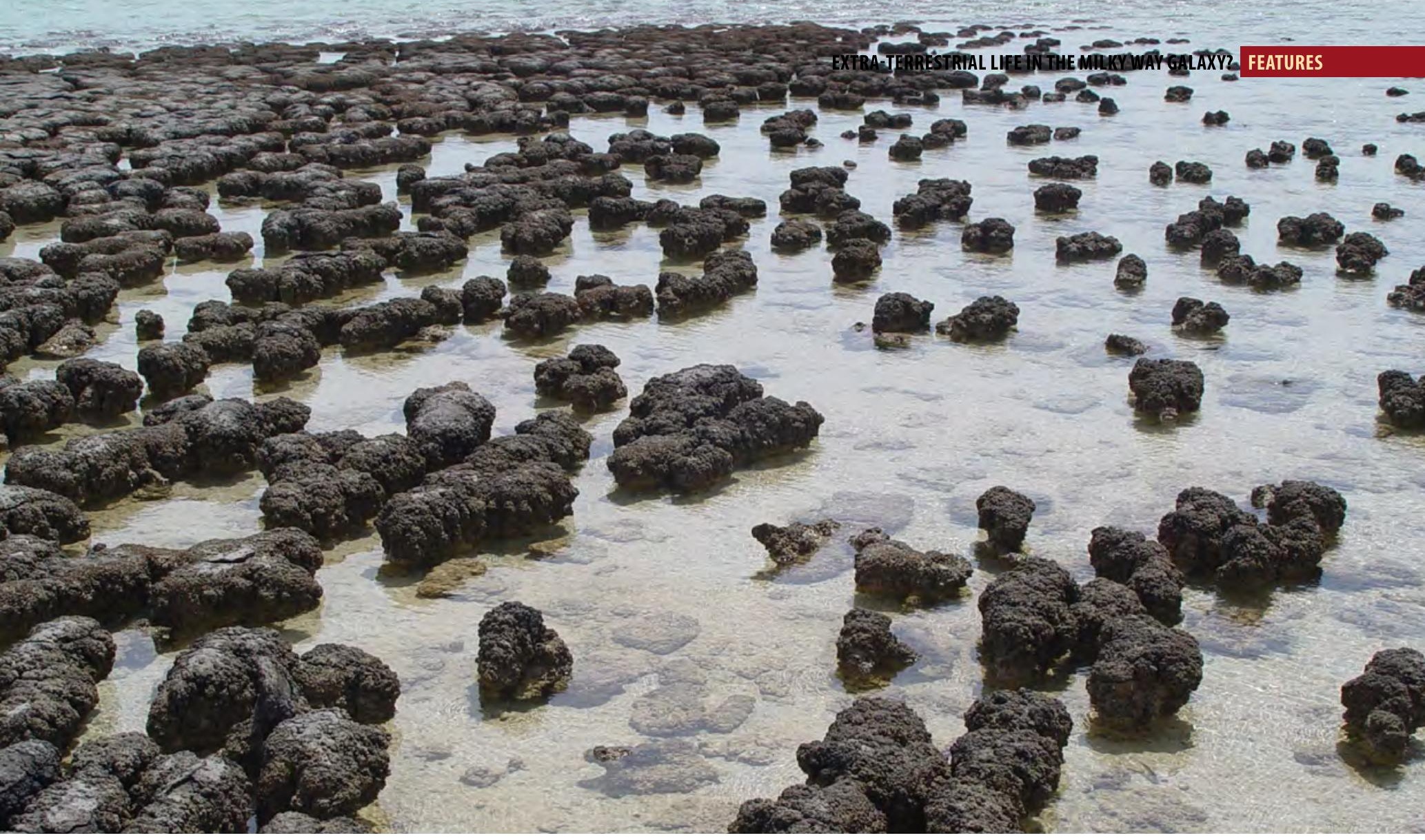

theory for how this happened. Even if a convincing model for the origin of life on Earth eventually emerges, it will be difficult to extrapolate to other planets to compute $f_{l}$. However, if clear evidence can be found for extraterrestrial life anywhere, we could at least be sure that $f_{l}$ is not vanishingly small. Consequently, it is tempting to search for signs of life outside the Earth. The most obvious target is Mars. Although searches for microbial life by the Viking landers in the 1970s turned up no convincing evidence, we now know that any life (or molecular fossils of early, but now extinct life) would need to be buried under about a metre of soil to avoid degradation by highly reactive molecules on the surface. A series of increasingly sophisticated probes have found strong evidence for sub-surface water. A probe using newer techniques to search for past of current life in samples excavated from deeper soil levels could be sent within a decade [3]. To ensure that any life on Mars arose independently, terrestrial contamination must be convincingly disproved. Missions to penetrate the ice and search for life in the sub-surface oceans of Europa have been considered, but they are more challenging.

To extend the search for life beyond our solar system, we could search for biomarkers in the atmospheres of planets around other stars. The oxygen in the Earth's atmosphere arose from photosynthetic activities by past life, and the current co-existence of oxygen and methane is considered a sign of current life. Studies of atmospheres of planets that transit (pass in front of) their stars have begun, but atmospheric studies of smaller, Earth-like planets are extremely challenging and will require a new generation of telescopes and instruments. At this point, possible values for $f_{l}$ range from very small $\left(e . g ., 10^{-9}\right)$ to unity.

\section{Intelligence}

Moving on to $f_{i}$ leads us into evolutionary biology, where once again, we have only our terrestrial experience to guide us. The facts are fairly clear. Life arose between 3 and 4 billion years ago and remained strictly prokaryotic, unicellular life ('microbial' for short) for most of Earth's history (Fig. 2). Multicellular, differentiated life appears between 500 and 600 million years ago and diversified in the Cambrian explosion beginning around 543 million years ago. Most of today's phyla, including ours (Chordata) trace to that period.

An interesting feature is that the timescale to evolve human level intelligence through biological evolution $\left(5 \times 10^{9}\right.$ years $)$ is comparable to the timescale for stellar evolution. If our evolution to intelligence has been twice as fast as on other planets, no other planets with life would have developed complex life. The likely cause of the long period was the need for microbial photosynthesis to produce an oxygen-rich atmosphere. A still more fundamental question is whether intelligent life with the characteristics needed for the next stage (technology) is a likely outcome on another planet. A diverse and changing environment is often invoked to select for intelligence, and it may have played a role in the evolution of Homo sapiens from a diverse array of hominids over the last few million years.

As with $f_{l}$, evidence for intelligent life on another planet would provide some constraints on $f_{i}$. Unfortunately, prospects for doing this are slim. Complex, non-technological life does not seem to produce any obvious atmospheric biomarkers. $\triangle$ FIG. 2: Currently living stromatolites in Australia. These are microbial mats of cyanobacteria, anaerobic organisms whose waste product of oxygen made more complex life possible. The oldest certain evidence of life is a fossilized stromatolite about 3.5 billion years old. 
v FIG. 3:

Communication

with extraterrestrial advanced civilisations

is usually assumed to use radio

wavelengths. Radio

telescopes can be

quite large as their

requirements on

surface accuracy

are much lower

than for telescopes

operating at visible

wavelengths. This

telescope at the

Dwingeloo Radio

Observatory is 25

metres in diameter.

After retirement from active astronomy use,

it has become a Dutch

industrial heritage

monument. It has

been refurbished by

volunteers and there

is some discussion

about using it to

search for signals

from extraterrestrial

civilisations.

\section{Communication capability}

Now we come to $f_{c}$, which assumes we have human level intelligence and is the fraction of planets with that level of intelligence that become "communicable." Since tool use is essential, purely aquatic planets that might have dolphin or whale intelligence probably need to be rejected at this point. While no such water worlds exist in our solar system, there is evidence for some around other stars. As long as we focus on stone-age or metal-age technology, we have several independent "experiments" on Earth. While cultural diffusion confuses the issue for the Africa-Eurasia land-mass, the Americas and Australia/New Guinea provide reasonably isolated cultures. The basis for all technological cultures is the development of agriculture, which allowed settled lifestyles, surpluses, and specialization of roles. It also triggered written language (for business records and taxes), metal extraction from ores, and observational astronomy for calendrical purposes. All these appear to have developed independently at least several times, providing some evidence that $f_{c}$ is not very small. Of course, no evidence exists for independent origins of higher technology because of the rapid diffusion of such technology around the world.

To engage in interstellar communication, a society must understand the nature of the Universe. For almost all of "Western" intellectual history, the Universe was conceived as geocentric and stars were assumed to be different from the Sun. While this was less true in some other cultures, none developed a correct world-view until the chain of events known as the Copernican Revolution proceeded to a reasonably correct understanding of the Milky Way in the early $20^{\text {th }}$ century. The connections between evolving worldview and technology are intriguing. The telescope played a major role in worldview evolution, while the Galilean and Newtonian revolution in physics was stimulated in part by trying to understand the cosmos. One can argue that technology and worldview (capability and interest in the Drake equation) are coupled, but one can perhaps imagine advanced civilizations on cloudy planets with incorrect worldviews.

The timescale for technology to develop is negligible compared to that for biological evolution. Advanced technological civilizations may produce planet-wide signatures. Ours has introduced novel chemicals, such as CFCs, into the atmosphere, for example. On one of the passages near Earth of the Galileo spacecraft as it was being boosted for its journey to Jupiter in 1990, its instruments were pointed at the Earth. Even with a resolution of $1 \mathrm{~km}$, the cameras found no clear indications of technological civilization; in contrast the radio emission from the Earth provided unambiguous evidence of advanced technology[4]. While no existing radio telescopes on Earth could detect current levels of this "leakage radiation" from extrasolar planets, it would not be prohibitively expensive to build such a telescope. However, the transition to fiber and digital transmission may mean that "radio-loud" planets are short-lived.

\section{Lifetime of Civilizations}

Once a communicable civilization arises on a planet, how long does it last? On this question, even our one example that we have used for the previous three factors fails us; we are still here! Technically speaking, a civilization needs only to lose interest in communicating to limit $L$,

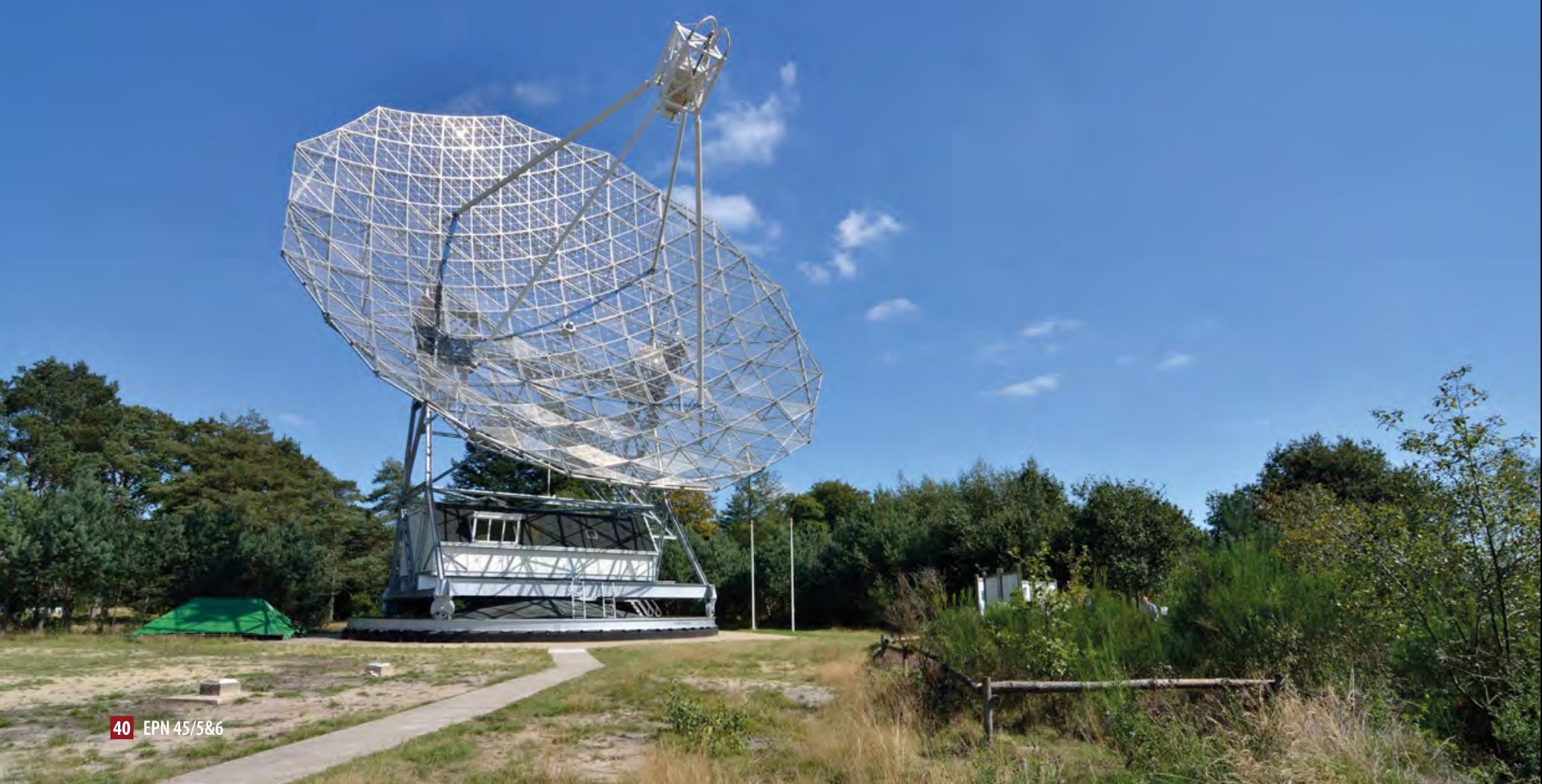


but it is more interesting to consider what might end the civilization altogether. We can divide the threats to our civilization to human-caused and natural threats. The human-driven threats all arise from the nature of technological civilizations, broadly conceived. Agriculture allows population growth, which leads to resource depletion and pollution. The main resource depletion issue is energy, actually low-entropy sources of energy. While the timescale for fossil fuel depletion is uncertain and contentious, it is probably measured in centuries, after which time, solar energy in various forms provides the only truly long-term source, with nuclear as an important bridging technology. Burning fossil fuels of course increases the greenhouse effect, which may provide some brake on fossil fuel use, but not enough to extend the reserves very much longer. The most apocalyptic possibility is all-out nuclear war, with arsenals like those of the US and USSR during the height of the cold war. Such an exchange could lead to "nuclear winter," causing crop failures and famine. Any survivors might well be disinclined toward, or incapable of, redeveloping such technology. It seems likely that either civilization will collapse or that a new, long-term worldview will emerge within centuries to millennia.

The most immediate natural threat is impact by asteroids or comets, as the recent Siberian incident has reminded us. There are dedicated efforts to find and catalog Near Earth Objects; with sufficient notice, we could modify the orbits of dangerous asteroids. Large objects, like the asteroid that may have finished the extinction of dinosaurs, have timescales of about $10^{8}$ years. The only truly certain natural threat is the evolution of our Sun. While the main sequence of a star is relatively stable, the star increases slowly in luminosity. In about 1 billion years, the rising luminosity will cause the loss of our oceans. In about 5 billion years, when the Sun becomes a red giant, the Earth is likely to spiral into the Sun. The only escape will be to send some people to a habitable planet around another star. Possible values for $L$ range from decades to 5 $\times 10^{9}$ years. A few long-lived civilizations will completely dominate the average lifetime. This logic indicates that the civilizations we might communicate with are very likely to be much older and presumably more advanced. (The latter presumption depends on an assumption of continued progress, which is debatable.) The fact that our searches have listened for, rather than broadcasted, messages is based on the conclusion that we are the less advanced civilization. If we mark our emergence by our first attempt to listen for a signal, that was about 60 years ago, so we would be one of the youngest civilizations in the Galaxy.

Armed with all the estimates, anyone can calculate his or her own estimate for $N$.

If none of $f_{l}, f_{i}$, or $f_{c}$ is very small, the answer will depend primarily on the choice of $L$. Of course, the ultimate test of any estimate of $N$ would be to detect signals from an extraterrestrial civilization (Fig. 3). The Milky Way

\section{THE DRAKE EQUATION}

The number of civilizations in our Galaxy with which we could communicate is given by

$$
N=R_{*} f_{p} n_{e} f_{l} f_{i} f_{c} L, \text { where }
$$

$R_{*}$ is the rate of star formation,

$f_{p}$ is the fraction of stars with planetary systems,

$n_{e}$ is the number of habitable (Earth-like) planets per planetary system,

$f_{l}$ is the fraction of habitable planets where life begins,

$f_{i}$ is the fraction of planets with life that evolves to a level of intelligence comparable to that of humans,

$f_{c}$ is the fraction of those that develop a technological civilization with the capability and interest in interstellar communication, and

$L$ is the lifetime of such civilizations.

The first three factors are astrophysical, while $f_{l}$ lies at the transition from chemistry to biology, $f_{i}$ is biological, and the last two factors involve history and predictions about technological developments and social interactions. The value of the Drake equation is in organizing our thinking rather than in providing an answer.

"society" will be dominated by the civilizations that develop the "long-view" before they destroy themselves.

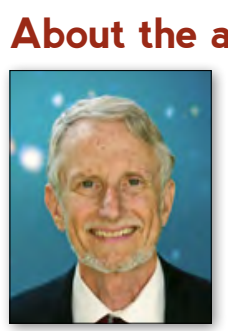

uthor

Neal Evans obtained his doctorate in physics at the University of California, Berkeley working with Nobel laureate Charles Townes. Evans joined The University of Texas at Austin faculty in 1975, where he teaches several astronomy courses, including a class about extraterrestrial life. Evans has led large programs on both the Spitzer and Herschel space telescopes, studying the formation of stars and planet-forming disks. He is currently the Edward M. Randall, Jr., MD Centennial Professor at the University of Texas at Austin and the Oort Professor for 2014 at Leiden University.

\section{References}

[1] R. Heller and J. Armstrong, Astrobiology 14, DOI: 10.1089/ast.2013.1088 (2014).

[2] S. Seager, Science 340, DOI: 10.1126/science.1232226 (2013).

[3] C.P. McKay and V. Parro Garcia, Scientific American 310-6, 44 (2014)

[4] C. Sagan et al., Nature 365, 715 (1993)

\section{SUGGESTION FOR FURTHER READING:}

Planets and Life, ed. Sullivan and Baross, Cambridge Univ. Press (2007). 